\title{
L'Europeizzazione del mondo e il suo declino
}

\section{La sovrastante potenza dell'Europa e la combinazione dei suoi fattori}

Quando si guarda a Roma nel periodo compreso tra la fine delle guerre puniche e la morte di Costantino - nel corso del quale essa andò affermando tutta la sua potenza, che la mise in grado di conquistare in misura sempre maggiore, assoggettare e ordinare quello che ai suoi occhi era tout court il mondo - è dato vedere come i fattori di questa potenza, che le conferì una posizione di forte superiorità rispetto ai soggetti politici, sociali e istituzionali con i quali Roma entrò gradualmente in urto, costituissero un unicum, un qualcosa senza riscontri nella scena geopolitica formata dai territori compresi nell'impero. Questo unicum era la combinazione di una cultura politica e giuridica, di un'architettura istituzionale che dal centro si articolava a livello delle province, di un governo disciplinatore sia delle parti sociali collocate nel nucleo dominante sia delle molteplici popolazioni organizzate gerarchicamente all'interno dell'impero in espansione, di un alto livello di capacità produttiva e di conoscenze tecnologiche, di strutture militari dotate di una efficacia offensiva e difensiva in grado di garantire un'incontrastata supremazia, di una efficiente rete di comunicazioni intese a favorire gli scambi commerciali e la dislocazione delle forze armate preposte ad assicurare la difesa all'interno dell'ordine costituito, la protezione dei confini e la loro dilatazione. Questo fu dunque il convergere di fattori che rese possibile la romanizzazione del mondo antico in Occidente fino alla sua crisi e poi al crollo definitivo.

Quella “combinazione di potenza” che aveva caratterizzato Roma dominatrice scomparve nella sua forma piena dalla scena del Vecchio continente per oltre mille anni. Essa si protrasse o si riprodusse, ma in una maniera soltanto parziale in ambiti territoriali limitati, nell'impero bizantino, nell'impero islamico dell'epoca d'oro, nell'impero ottomano, in Persia, nell'India del Gran Mogol: imponenti costruzioni politiche e istituzionali, dotate bensì di capacità di dominio, di forza ordinatrice e anche di un dinamismo espansionistico di grandissimo vigore, tutte però prive della forza risolutiva che aveva consentito a Roma di acquisire ecco il punto - un incontrastato monopolio di potenza. Neppure l'impero cinese poté sostenere il paragone, a causa delle ondate di gravissime turbolenze e divisioni interne, delle ricorrenti convulsioni intestine, della lotta delle etnie per la prevalenza, dei conflitti tra le dinastie. 
La combinazione tornò a riproporsi in Europa tra il Settecento e la seconda metà dell'Ottocento in una forma - rispetto al modello romano - per un verso assai simile, per l'altro assai differente: anzi del tutto contrastante. La somiglianza era data dai molteplici e importanti elementi di unità; il contrasto da una frammentazione di Stati che si accompagnava a continue guerre intestine.

Come Roma, anche l'Europa aveva acquistato una forte identità che ne segnava la diversità rispetto al resto del mondo. Essa era caratterizzata nel suo caso specifico dalla comune matrice cristiana, seppure dilaniata dalle contrapposizioni tra cattolici, protestanti e ortodossi; da una cultura politica, giuridica, letteraria e scientifica che, al di là delle molteplicità e diversità delle sue correnti ed espressioni, costituiva un patrimonio comune delle sue élites; da forti istituzioni statali; da un'economia in espansione cui il processo di industrializzazione a partire dagli ultimi decenni del XVIII secolo impresse un crescente dinamismo; da progressi senza eguali nel campo del sapere scientifico e delle applicazioni tecnologiche; da una sovrastante potenza dei suoi apparati militari rispetto al resto del globo. Furono questi gli elementi che permisero all'Europa di acquisire una superiorità a mano a mano maggiore nei confronti dei grandi imperi ottomano e asiatici, che tra Cinque e Seicento erano ancora stati in grado di contrastare quella superiorità. E fu quest'ultima che consentì all'Europa di dare - dopo il primo grande ma pur sempre parziale "assalto" in direzione degli altri continenti a iniziare dalla fine del Quattrocento con la conquista delle Americhe - un rinnovato e ancor più vigoroso assalto al resto del mondo e di imporre quella che venne chiamata la sua "centralità", destinata a durare sino alla prima guerra mondiale. Occorre però notare che tale centralità mostrò i primi segni di significativo indebolimento nella seconda metà del XIX secolo in conseguenza del rapido rafforzamento economico e politico degli Stati Uniti e degli inizi della modernizzazione istituzionale e militare del Giappone.

Ne Lo spirito delle leggi Montesquieu espresse compiutamente la coscienza che gli europei avevano maturato della propria posizione dominante. Due affermazioni riescono in proposito particolarmente illuminanti: l'una riguarda la posizione acquisita dal Vecchio continente nel mondo, l'altra quella tenuta da alcuni paesi nel continente stesso. Montesquieu prima scrive che "l'Europa ha raggiunto un grado così elevato di potenza da non trovar riscontro nella storia”; poi, con riferimento specifico agli indici probanti di maggior dinamismo al suo stesso interno, nota che, se "l'Europa svolge il commercio e i traffici marittimi delle altre tre parti del mondo", Francia, Inghilterra e Olanda "svolgono quasi tutto il commercio e la navigazione dell'Europa”. Egli offriva così una chiave decisiva per comprendere per un verso le caratteristiche più generali del rapporto tra l'Europa e il resto del mondo e per l'altro la gerarchia stabilitasi in campo economico tra i paesi in prima fila e quelli in seconda o terza fila. Un altro elemento 
che contribuiva a conferire all'Europa la sua netta superiorità Montesquieu lo individua negli effetti della rivoluzione scientifica che, con radici riconducibili al Quattro-Cinquecento, era andata dispiegandosi con vigore crescente a partire dal Seicento. In una delle Lettere persiane egli fa dire allo stupito Usbek che in Europa vi sono filosofi che "seguono in silenzio le tracce della ragione umana" e che non vi è da credere "fino a dove li ha condotti questa guida. Essi hanno decifrato il Caos, e con una semplice meccanica hanno spiegato l'ordine dell'architettura divina”.

$\mathrm{Fu}$ anche Voltaire nel Saggio sui costumi e lo spirito delle nazioni a sottolineare come il convergere di cultura e progressi scientifici e tecnologici avessero conferito all'Europa strumenti di potere quali non si erano mai avuti in epoche precedenti e non si davano in altre zone del mondo. Ma la notazione in lui più tipica fu l'insistere, come si vede bene ne Il secolo di Luigi XIV, sul fatto che "nonostante le guerre, e nonostante le diversità di religione", era andata stabilendosi una "repubblica letteraria”, una "grande società degli spiriti" che legava gli intellettuali mediante un comune linguaggio culturale e civile. Si trattava di un Leitmotiv condiviso, al di là di tutte le loro personali diatribe, da Rousseau; tanto che il primo poteva affermare che l'Europa era unita dagli "stessi principi politici e di diritto pubblico e di politica; ignoti nelle altre parti del mondo” e il secondo che in Europa era venuta costituendosi una "società di popoli”, la quale univa in un "sistema" operante "attraverso la stessa religione, il medesimo diritto delle genti, i costumi, le lettere, il commercio, e mediante una sorta di equilibrio che è la conseguenza necessaria di tutto ciò”.

All'incirca un secolo dopo, un altro grande intellettuale, l'inglese John Stuart Mill, nel suo celebre saggio Sulla libertà rifletteva - in un'epoca ormai pienamente investita dagli effetti del processo di industrializzazione che aveva più che mai il suo centro propulsore nel Vecchio continente - su "che cosa ha reso le nazioni europee un settore dell'umanità che si evolve e non resta statico" e quindi sulla loro "superiorità - che, quando esiste, è un effetto e non una causa -". A spiegazione, faceva riferimento alla mancanza di uniformità, all'affermarsi di una molteplicità di caratteri e di correnti culturali, alla capacità di praticare "una gran quantità di vie", allo sperimentalismo, tal che "i tentativi reciproci di impedir il progresso altrui hanno raramente avuto un successo definitivo, e a lungo andare tutti hanno avuto la possibilità di recepire i risultati positivi altrui”. La conclusione suonava: “l’Europa deve a questa pluralità di percorsi tutto il suo sviluppo progressivo e multiforme”. Al che però Mill faceva seguire la significativa considerazione che occorreva domandarsi fino a quando essa sarebbe stata in condizione di godere di questa "dote”. 


\section{Il culmine della "centralità" europea tra Sette e Ottocento}

Voltaire e Rousseau parlarono di un'unità culturale e civile che era andata affermandosi in Europa nonostante le sue divisioni interne, i contrasti politici e religiosi e le guerre. Orbene, chi guardi alla storia del continente tra la fine del Quattrocento e l'Ottocento non fa fatica a vedere come, in contrasto con quegli elementi di unità, essa fu segnata da ricorrenti ondate di violenze messe in atto dai maggiori Stati europei, che, dotati di imponenti apparati militari, tentarono, singolarmente o con la loro rete di alleanze, di assumere - nella forma sia del dominio diretto sia dell'egemonia - una posizione di primato: tentativi tutti infine falliti dopo successi solo parziali e temporanei. Giusto quindi il detto di Kant che le paci raggiunte tra le potenze europee, dando una sostanza precaria ai periodi di "equilibrio" da queste convenuti, risultavano unicamente "tregue" tra una guerra e l'altra. I più consistenti tentativi di stabilire il predominio nel periodo sopra indicato furono in successione quelli nella seconda metà del Cinquecento di Filippo II, nella prima metà del Seicento degli Asburgo d'Austria e di Spagna, a cavallo tra Sei e Settecento di Luigi XIV, a cavallo tra Sette e Ottocento della Francia rivoluzionaria e napoleonica; tentativi cui seguì infine nella seconda metà del XIX secolo quello culminato negli scontri della Germania bismarckiana prima con l'Austria e poi con la Francia di Napoleone III conclusisi con l'emergere della prima a maggiore potenza dell'Europa continentale. In questo quadro la Gran Bretagna - che, resa straordinariamente forte a partire dalla rivoluzione industriale di cui fu il centro irradiatore e il nucleo più vigoroso, con l'effetto di consentirle uno sviluppo commerciale e industriale senza confronti per circa un secolo - tenne costantemente parte a sé. Essa era tesa a preservare e a difendersi dalle invasioni esterne, a far valere tutto il peso della sua incontrastata prevalenza navale, a spostare la propria forza nei conflitti tra gli Stati europei avendo quale fine costante di impedire che nell'Europea continentale l'intento di questa o di quella potenza di acquisire il predominio assumesse il carattere di una duratura stabilità.

La storia d'Europa a partire da quelli che si considerano gli inizi dell'età moderna era stata la storia di grandi guerre tra le maggiori potenze, che avevano messo in sempre maggiore evidenza la crescente efficienza dei rispettivi apparati bellici. Sennonché nei secoli XVI e XVII questa efficienza non aveva ancora raggiunto nei confronti degli eserciti terrestri e delle flotte dell'impero ottomano e degli imperi asiatici un grado di incomparabilità: che venne invece raggiunta in maniera via via più accelerata nel corso del secolo XVIII. Fu allora che la bilancia a livello mondiale andò irresistibilmente pendendo a favore della maggiori 
potenze europee, le quali - dopo che Spagna e Portogallo erano entrate in una fase di crescente decadenza - si erano ridotte a quattro: la Gran Bretagna, la Francia, l'Austria e la Russia, la quale ultima era entrata nel concerto dei grandi Stati del continente grazie al processo di modernizzazione energicamente avviato da Pietro. Ad affacciarsi al loro fianco, era salita, ma ancora in una posizione di relativa minorità, la Prussia. Sennonché nel cerchio più ampio delle potenze europee era andato nettamente delineandosi un nucleo più ristretto. Il quale era composto da Gran Bretagna e Francia, i due paesi più sviluppati economicamente e più forti militarmente, i quali congiuntamente ma in maniera concorrenziale nella seconda metà del secolo diedero un fortissimo impulso - con il concorso secondario ma significativo della Russia e quello certo non trascurabile di altri Stati europei - a quello che si può definire l' "assalto al mondo". Procedevano così parallelamente i conflitti di potenza all'interno dell'Europa e la congiunta lotta per l'assoggettamento, nella duplice specie dell'acquisizione di influenza e della conquista, di parti crescenti dei continenti extraeuropei. Ora l'impulso all'europeizzazione del mondo conobbe una vigorosa accelerazione, nella forma del moderno imperialismo-colonialismo, che, mentre mostrava appunto la comune matrice europea, era messo in atto dai grandi Stati rivali. Venivano esportati e imposti - secondo le caratteristiche tipiche di ciascuna potenza - culture politiche, istituzioni giuridiche, apparati burocratici e modalità organizzative, tecniche produttive, merci, anche costumi, e stabilite le élites dominanti a cui era affidato il compito di esercitare il potere e il controllo sui territori assoggettati. Può dirsi che le frontiere dell'Europa, superando quelle propriamente geografiche, andarono via via allargandosi alle parti del mondo in cui questa estendeva la propria influenza nei suoi molteplici aspetti.

La "centralità" europea raggiunse il suo culmine tra la seconda metà del XVIII secolo e la prima metà del XIX. Allora essa fu piena e incontrastabile, dopo di che si protrasse ancora per poco più di sessant'anni; ma - come si è già osservato - vide però levarsi contro la sua superiorità a Occidente la potenza produttiva degli Stati Uniti, divenuti agli inizi del Novecento la maggiore singola economia a livello mondiale e a Oriente il "risveglio" del Giappone: potenza degli uni e risveglio dell'altro entrambi - si badi - tributari dell'influenza dell'Europa e dell'importanza dei rapporti con essa, ma anche espressioni della volontà di mettere ormai in discussione una supremazia che non poteva più essere quella di un tempo. Gli Stati Uniti erano per molteplici e determinanti aspetti figli dell'Europa, che aveva loro trasmesso cultura, essenziali punti di riferimento politici e istituzionali cui ispirarsi e con i quali confrontarsi, scienza e tecnologia; e sotto questo aspetto la loro storia costituiva un capitolo significativo dell' "europeizzazione del mondo". Ma al tempo stesso fin dalle origini del loro costituirsi in Unione, del formarsi delle loro istituzioni politiche e sociali e della elaborazione di un loro proprio 
"spirito nazionale", si erano posti su posizioni persino aspramente critiche nei confronti della "madre Europa”. Si pensi in proposito al dibattito sulla Costituzione e in particolare agli articoli di Hamilton e di Madison nei Federalist Papers, dove la madre appariva per molti versi come un contromodello e una "matrigna": che intendeva dominare il mondo, imporre i propri interessi su quelli altrui, che, nella varietà delle scene offerte dai suoi molti paesi, dava deplorevoli esempi di regimi dispotici, di avversione ai principi di libertà degli individui e delle collettività, di abissali iniquità economiche e sociali, di rigide barriere di classe, di guerre tra le opposte parti della società e tra gli Stati. Lo spirito degli americani fu sin dalle origini quello di figli ribelli, che - specie dopo la guerra civile che ne rinsaldò fortemente il vincolo unitario - diedero vita ad una comunità capace sia di una crescente autonomia dall'Europa sia di dotarsi di una cultura dai tratti originali sia di intraprendere la strada di un accelerato sviluppo economico che affiancava un apparato industriale in espansione ad una possente agricoltura sia di diventare la terra pronta ad accogliere milioni di poveri e migliaia di rifugiati politici desiderosi di fuggire dalla durezze del Vecchio mondo. Fu così che gli americani giunsero a concepire, mediante un processo di rovesciamento, un senso di decisa superiorità non solo più morale ma ormai anche materiale nei confronti di quello.

Pressoché in parallelo corse il risveglio del Giappone, il paese che nella prima metà del XIX secolo dovette far fronte, seppure in forme meno umilianti e drammatiche della Cina, alle pretese e ingiunzioni per un verso di russi, inglesi e olandesi e per l'altro degli americani. Furono in particolare questi ultimi, arrivati nel 1846 e soprattutto nel 1853 con le loro navi, a umiliare maggiormente i giapponesi, a renderli consapevoli della loro incapacità di resistere. Il che li indusse, per reazione, ad avviare quel processo di modernizzazione economica, istituzionale e militare che nel giro di meno di quarant'anni li avrebbe messi in grado di sconfiggere nella guerra del 1904-1905 la Russia. Fu però ancora un segno del prestigio e dell'influenza dell'Europa il fatto che il governo imperiale nipponico, per apprendere le vie della propria modernizzazione e formare i quadri ad essa necessari, si rivolgesse alla Gran Bretagna, alla Francia e alla nuova Germania. Ma segno dei tempi era che gli apprendisti andassero a scuola dei loro insegnanti europei per sottrarsi quanto più rapidamente possibile alle imposizioni dei bianchi del Vecchio Mondo (e anche del Nuovo). La potenza ormai dispiegata e destinata ad una sempre maggiore ascesa degli Stati Uniti e del Giappone costituì la testimonianza dell'inizio della crisi della centralità di quell'Europa che per circa due secoli si era eretta a padrona del globo e largamente lo era stata: centralità che sarebbe letteralmente naufragata nel 1914-1918, quando apparve che sì nel corso del suo predominio essa aveva diffuso al di là dei propri confini cultura, istituzioni, scienza e tecnica, ma contemporaneamente aveva insegnato ad altri 
paesi come impadronirsi della potenza che aveva a lungo costituito un monopolio europeo e come da ultimo spezzarla, ponendo così fine all' “europeizzazione del mondo".

\section{Tappe dell'ascesa e del declino della centralità dell'Europa nel mondo moderno}

Punto decisivo della fase ascendente della centralità dell'Europa nel mondo moderno fu costituito dalla cosiddetta "Guerra dei sette anni", che, dispiegatasi tra il 1756 e il 1763, ebbe il carattere di un grande conflitto combattuto sia all'interno del Vecchio continente sia nelle Americhe sia in Asia. Essa mostrò appieno come la potenza europea si manifestasse al tempo stesso nelle guerre tra i suoi Stati e nella spinta espansionistica esterna di cui furono allora protagoniste la Gran Bretagna e la Francia; e a quale grado di forza e di efficienza fossero giunte le macchine belliche europee, le uniche ormai capaci di confrontarsi reciprocamente. Si trattava di una superiorità che sarebbe stata ulteriormente ed enormemente accresciuta dagli effetti della rivoluzione industriale. La Guerra dei sette anni fu quella che si può definire propriamente la prima guerra mondiale dell'età moderna, in quanto combattuta su tre continenti. Le sue conseguenze più importanti furono da un lato il riconoscimento e il consolidamento della Prussia quale nuova potenza europea e dall'altro la posizione preponderante acquistata in campo coloniale dalla Gran Bretagna, che distrusse l'impero francese nell'America settentrionale ed eliminò le posizioni acquisite della Francia in India, dove consolidò un'autorità destinata ad estendersi e a affermarsi sempre più ampiamente e profondamente. Sicché tanto nell'America del Nord quanto in India l'europeizzazione assunse il volto di una "europeizzazione inglese”.

Occorre a questo punto notare che il fenomeno di enorme portata che definiamo europeizzazione del mondo ebbe per un verso un volto bensì unitario in relazione ai tratti comuni che la civiltà europea nelle sue componenti presentava, ma per l'altro verso quello impresso nel loro rapporto con le altre parti del mondo dalle molteplici specificità giuridiche, economiche, culturali, ideologiche e anche religiose dei singoli Stati colonialistici e imperialistici. Nel periodo in cui l'Europa nel suo insieme fece valere al massimo la sua potenza e la sua centralità, si ebbe quindi una pluralità di "europeizzazioni”: al primo posto vi fu l'inglese, al secondo la francese, seguite da quella olandese e belga, per limitarci a segnalare le maggiori. Sotto questo profilo nel Sette-Ottocento queste europeizzazioni si posero in continuità con quelle di cui nei secoli precedenti erano state la spagnola e la portoghese. 
Si è detto che la più importante delle molteplici europeizzazioni fu l'inglese. E infatti essa si dispiegò in tutti i continenti extraeuropei nella forme vuoi dell'assoggettamento diretto, vuoi delle formazioni giuridiche e politiche che sarebbero culminate nel Commonwealth basato su principi di relativa autonomia, vuoi dell'egemonia stabilita su Stati sottoposti alla sua influenza politica ed economica. Fu un processo che portò alla creazione di un gigantesco impero che per ampiezza territoriale e profondità della penetrazione non aveva precedenti nella storia. Di peso grandissimo fu anche l'europeizzazione francese, che si estese anch'essa - con una progressiva accelerazione nel secolo XIX a partire dalla conquista dell'Algeria nel 1830 - in tutti i continenti extraeuropei; mettendo in evidenza la prevalente volontà di assimilazione diretta dei territori d'oltremare nell'ambito della "più grande Francia”.

È un dato acquisito che aspetto comune, seppure manifestato in gradi diversi, della centralità dell'Europa fu la costruzione dell'ideologia della superiorità degli europei; che, ancora una volta, aveva i suoi precedenti nei più vecchi imperi di Spagna e Portogallo. In essa, intesa a dare una legittimazione ai vari atti di conquista, all'esercizio del dominio e al diritto degli europei a fare valere la loro guida sui popoli "inferiori” del mondo, si intrecciavano, obbedendo sia a convinzioni sia a intenti meramente strumentali, morale, religione, diritto, pseudoscienza. Il razzismo costituì l'espressione più cruda e vistosa di quella costruzione.

Bisogna d'altra parte sottolineare che l'europeizzazione del mondo non si espresse unicamente con il colonialismo e l'imperialismo, con la conquista territoriale, il duro e persino crudele assoggettamento dei popoli giudicati inferiori. Essa ebbe altresì aspetti che possiamo senza esitazioni definire "progressisti". Fu dall'Europa che gli altri continenti presero le più avanzate conoscenze scientifiche e le tecnologie prodotte dalla rivoluzione industriale e dai suoi sviluppi. Può dirsi che il treno e la nave a vapore furono forse i maggiori simboli dell'europeizzazione modernizzatrice del mondo. Dal Vecchio continente vennero anche le grandi correnti culturali, i modelli istituzionali e politici e i miti ideologici che diventarono patrimoni importanti e decisivi per la formazione delle élites degli altri continenti. Dall'Europa si diffusero nelle altre parti del globo, con effetti suscettibili di diverso giudizio, illuminismo, idealismo, positivismo, materialismo storico, liberalismo oligarchico-conservatore e liberalismo democratico, nazionalismo democratico e nazionalismo autoritario, neocesarismo, socialismo e comunismo.

Si sono già sottolineate, a proposito del rapporto tra questa e il resto del mondo, l'importanza e l'originalità del caso degli Stati Uniti. La nascita degli Stati Uniti venne salutata da numerosi intellettuali e ideologi europei come una sorta di nuovo inizio del mondo e come annuncio di una libertà destinata a uscire dai loro confini. La celebrarono con toni entusiastici grandi poeti come Byron, 
Shelley e Goethe, che si trovarono concordi nell'esaltare la giovinezza prometeica degli americani rispetto alla vecchiezza degli europei. Ma più dense di intelligenza storica furono le considerazioni di un Hegel e di un Cousin; il primo dei quali nelle Lezioni sulla filosofia della storia, tenute tra il 1821 e il 1831, parlò dell'America come del "paese dell'avvenire", e il secondo nella Introduzione alla storia della filosofia del 1845 di un potente soggetto emergente candidato a raccogliere la successione dell'Europa. Dal canto suo Tocqueville nella prima parte de La democrazia in America, pubblicata nel 1835, in un passo celeberrimo - prefigurando un'Europa (il riferimento era palesemente all'Europa ad occidente della Russia) ancora padrona della terra ma destinata ad andare incontro a un processo di decadenza - profetizzava l'ascesa parallela dei russi e degli americani, che "entrambi sembrano chiamati da un disegno segreto della Provvidenza a tenere un giorno nelle loro mani i destini di metà del mondo”, servendosi gli uni dei mezzi offerti dalla servitù e gli altri di quelli della libertà. Fu Tocqueville a diffondere nella cultura europea quest'idea della futura grandezza del colosso americano e di quello russo. Ma essa non era di per sé originale. La troviamo, ad esempio, già nettamente formulata nelle parole dello slavofilo russo Kireevskij, il quale nel 1829 contrapponeva alla corrodente vecchiezza dell'Europa occidentale il futuro di grandezza che attendeva l'America e la Russia, i cui popoli erano "giovani e non logorati”.

Quando vennero avanzate, le previsioni e profezie degli Hegel, dei Cousin, Kireevskij e Tocqueville apparivano ed erano, in relazione allo stato delle cose, mere ipotesi, frutti dell'immaginazione. Erano al tempo stesso testimonianze, seppure ancora assai minoritarie, della consapevolezza - in palese e netta controcorrente rispetto al senso di una intangibile sicurezza nella superiorità dell'Europa la quale continuava a caratterizzare il pensiero e i sentimenti prevalenti delle sue élites intellettuali e politiche - che il primato dello stesso nucleo costituito dai paesi più vigorosi e dinamici del Vecchio continente doveva essere visto alla luce dei cicli che avevano e avrebbero scandito la storia universale. Come si è detto, quelle erano ancora ipotesi, immaginazioni; e infatti la centralità dell'Europa e la sua capacità di influenza e di dominio nel mondo non avevano allora neppure raggiunto l'apice. Questo venne infatti toccato nel ventennio tra gli anni Cinquanta e Ottanta dell'Ottocento dall'Europa capitalistica e borghese, postasi alla testa di un processo di modernizzazione economica che non aveva eguali, dotata di una potenza finanziaria, di apparati industriali, di una rete di comunicazioni e capace di una penetrazione commerciale senza pari: una superiorità che fece da impulso ad una ulteriore fase della conquista e della colonizzazione di nuovi immensi territori. Le Esposizioni universali di Londra del 1851 e di Parigi del 1855 e del 1889 furono le grandiose vetrine della persistente superiorità 
europea, pur prossima in quest'ultima data ad essere ormai sfidata in campo economico dall'impetuosa ascesa degli Stati Uniti.

\section{Crisi e crollo della centralità europea}

La spinta espansionistica che portò le potenze europee negli ultimi decenni che precedettero il 1914 a dilatare ulteriormente, e sovente in reciproco contrasto, le frontiere di imperi già immensi fu insieme lo specchio da un lato della loro persistente comune potenza e dall'altro delle loro debolezze e fratture; le quali si sarebbero pienamente dispiegate, rivelandosi catastrofiche, durante la prima guerra mondiale e nel dopoguerra. Il periodo di pace intercorso tra la guerra franco-prussiana e l'inizio del grande conflitto aveva solo malamente mascherato le fratture. Infatti, la tregua durata poco meno di mezzo secolo aveva visto il riaccendersi della rivalità tra la Francia e la Germania unificata; il balzo in avanti di quest'ultima trasformatasi con una crescente intensità in un paese industriale in grado di sfidare e superare la Gran Bretagna per capacità di organizzazione dei fattori produttivi, modernità degli impianti e dinamismo commerciale, e di costruire la più efficiente e possente macchina militare del mondo; il consolidarsi infine degli schieramenti formati per un verso dalla Triplice Intesa tra Gran Bretagna, Francia e Russia e per l'altro dalla Triplice Alleanza fra Germania, Austria e Italia. L'esito fu prima l'esplosione delle antitesi e poi il crollo della centralità europea. Il fatto del tutto nuovo fu che - mentre nel corso dell'età moderna l'Europa era stata bensì la sede di grandi guerre intestine susseguitesi in continue ondate, ma queste non avevano mai messo in discussione la sua complessiva superiore potenza economica, tecnologica e militare, che era andata anzi costantemente aumentando, costituendo il presupposto dell'europeizzazione del mondo - ora si arrivò alla fine di quel ciclo storico.

La crisi della centralità europea aveva avuto, come si è già avuto modo di ricordare, le sue eloquenti avvisaglie nella vertiginosa ascesa economica degli Stati Uniti e in quella parallela del Giappone, dotatosi, oltre che di un ragguardevole apparato produttivo, anche di possenti forze armate e nel fatto che entrambi i paesi erano entrati con slancio sulla scena delle contese imperialistiche. Ciò nonostante, prima del 1914 quella crisi si presentava pure sempre relativa, in quanto il Vecchio continente continuava a concentrare nell'insieme delle sue componenti la maggiore forza industriale, una soverchiante superiorità militare e il possesso o il controllo di sterminati territori extraeuropei. Ma, e questo costituiva un punto cruciale, a differenza che nei secoli precedenti, esso non era più in grado di comunicare al mondo esterno quell'impulso che abbiamo chiamato 
“progressista” costituito dall'espandersi della sua più avanzata cultura, della sua scienza e della sua tecnologia. Sempre maggiori zone del mondo esterno si erano rese e andavano rendendosi capaci di sviluppare risorse proprie.

Un tale profondo cambiamento non veniva ancora percepito nella sua importanza e nelle sue potenziali implicazioni dalle classi dirigenti dei maggiori Stati del Vecchio continente, rimaste chiuse in un inamovibile e arrogante senso di superiorità e rassicurate da una soverchiante potenza militare, ritenuta una invalicabile barriera da parte del resto del mondo. Tra gli intellettuali che per contro mostravano a quel punto piena coscienza dell'approssimarsi di una svolta epocale si trovava in prima fila Georges Sorel, il quale, quando ormai l'Europa andava correndo verso l'immane conflitto, affermò che un'intera stagione della sua storia si era chiusa, in quanto essa non aveva più nulla di grande da dare al resto del mondo e che - scriveva - "una sola idea unisce l'Europa” ovvero: "l’idea della guerra”.

Gli avvenimenti svoltisi in Europa nel corso della prima guerra mondiale tra il 1914 e il 1918 mostrarono, come se al vaso di Pandora che li racchiudeva fosse stato improvvisamente tolto il coperchio, tutte le divisioni e tutti i contrasti che erano andati maturando nel Vecchio continente, opponendo tra loro gli Stati e le nazionalità, le classi, gli schieramenti politici, le ideologie e i sentimenti. Nel crogiolo incandescente del conflitto, gli ultimi residui dell'originaria idea illuministica e prima ancora erasmiana della repubblica degli spiriti europei venne sepolta da una spaventosa, mai vista carneficina, dalle macerie materiali che distrussero l'economia del continente, in un contesto di odi furiosi condivisi dalle masse popolari e dalle élites culturali - al cui interno i celebratori della Kultur si contrapposero a quelli della Civilisation - e di scatenamento di una lotta senza quartiere dei nazionalisti imperialisti e autoritari contro i nazionalisti democratici e gli internazionalisti, dei socialisti fautori dell'abbattimento dello Stato borghese contro sia i socialisti moderati sia i liberali e sia i reazionari, dei movimenti anticapitalistici contro i difensori del capitalismo. Nell'apocalisse che produsse la sua autodistruzione l'Europa gettò nella grande fornace tutte le risorse umane, economiche e militari che aveva accumulato nel quarantennio della pace.

La fine della guerra ebbe conseguenze di portata storica universale. La più evidente di tutte fu il crollo definitivo della centralità europea. Infatti, la guerra spezzò irrimediabilmente quel moto ascendente dell'Europa che, iniziato nel XV secolo, venne ora drasticamente interrotto.

Nel corso della guerra si produssero cinque avvenimenti cruciali, il cui significato deve essere colto nei loro nessi reciproci. Il primo, il più clamoroso e simbolico, fu il fatto senza precedenti - si trattò del canto del cigno perverso della centralità dell'Europa - che le grandi potenze europee le quali avevano dato inizio al grande conflitto non erano state in grado di finirlo. Perché finisse, e in un certo 
modo, fu necessario che intervenissero nel 1917 gli Stati Uniti, erettisi a grande potenza oltre che economica anche militare. Fu infatti il loro apporto dapprima economico e finanziario e in seguito direttamente militare che soltanto consentì agli avversari degli Imperi centrali di resistere e infine di vincere. A esprimere la coscienza della svolta epocale erano le eloquenti parole indirizzate ai suoi compatrioti nel settembre 1919 dal presidente Wilson, con le quali rivendicava l'avvento di un nuovo primato: quello americano: "Nel volto dell'America si rispecchia il futuro e, miei concittadini, nel progetto dell'America si rispecchia il futuro del mondo". Era un modo per affermare chiaramente che il ruolo preponderante esercitato dai vecchi "lupi” europei negli affari internazionali apparteneva al passato.

Il secondo avvenimento fu il messaggio rivoluzionario universalistico lanciato da Lenin nel corso della guerra prima e dopo la presa del potere da parte dei bolscevichi in Russia nell'ottobre 1917, secondo il quale la vecchia Europa, in un quadro contraddistinto dal prorompere sulla scena di nuovi soggetti statali, nazionali, politici e sociali, era destinata a perdere il ruolo che aveva occupato in passato nella scena del mondo. Si era giunti al termine del secolare ciclo storico fondato sul primato nel mondo delle maggiori potenze europee e dei loro imperi, al quale andava sostituendosi quello della rivoluzione sociale e anticoloniale internazionale. Componente essenziale del discorso di Lenin era l'appello ai popoli coloniali, semicoloniali o comunque ancora soggetti agli Stati europei, alla rivolta contro i loro dominatori, invitati a unirsi a quella degli eredi di Spartaco che vivevano nei paesi più avanzati oppressi dallo sfruttamento capitalistico. Tanto Wilson quanto Lenin, pur da opposti punti di vista, convergevano nel registrare che la multisecolare centralità dell'Europa e quindi l'europeizzazione del mondo era andata incontro ad un crollo senza ritorno.

Il terzo avvenimento che nel dopoguerra fece da spia al crollo della centralità del Vecchio continente fu la distruzione della potenza della Germania, il cui impetuoso sviluppo economico, scientifico, tecnologico e militare era stato il dato più significativo della storia europea nel quarantennio precedente il 1914: potenza che agli inizi del secolo XX - quando pure gli Stati Uniti erano ormai ascesi al vertice della gerarchia mondiale in quanto più forte paese industriale - collocava quel paese, divenuto a sua volta il maggiore paese industriale in Europa, al primo piano nel mondo per l'eccellenza della sua macchina produttiva e per la capacità di far interagire organizzazione del lavoro, scienza e tecnologia. La "pace cartaginese" imposta da inglesi e francesi ai tedeschi nel 1919 costituì la tragica testimonianza che l'Europa, travolta dai suoi incomponibili conflitti intestini, brancolava in uno stato di completa cecità nella considerazione del proprio futuro: cecità manifestata dalla determinazione di Gran Bretagna e ancor più della Francia - vincitrici per interposta persona - di strangolare definitivamente la Ger- 
mania. Quale fosse lo spirito dominante dei capi francesi e inglesi, su cui gravava principalmente il compito della difficilissima rimozione delle macerie che seppellivano il continente, lo espresse con parole di fuoco indirizzate a Clemenceau e a Lloyd George Keynes nel suo celebre saggio Le conseguenze economiche della pace: “La vita futura dell'Europa non li riguardava; i suoi mezzi di sopravvivenza non davano loro alcuna ansietà. Le loro preoccupazioni, buone e cattive ad un tempo, si riferivano alle frontiere e alle nazionalità, all'equilibrio delle forze, agli ingrandimenti imperialistici, al futuro indebolimento di un nemico forte e pericoloso, alla vendetta e a riversare dalle spalle dei vincitori su quelle dei vinti gli insostenibili pesi finanziari”.

Il quarto avvenimento fu il crollo dell'impero zarista cui fece seguito l'ascesa al potere dei bolscevichi in Russia e il disfacimento dell'impero multinazionale austro-ungarico. Il primo ebbe l'effetto di scatenare dagli Urali alle coste dell'Atlantico una guerra ideologica, politica e sociale che per la violenza delle contrapposizioni aveva precedenti comparabili unicamente nelle guerre civili e religiose causate dalla Riforma protestante e nello scontro tra la Francia rivoluzionaria e napoleonica e il variegato e contraddittorio schieramento degli Stati ad essa ostili. La differenza era che quelle contrapposizioni e quei conflitti, pur tanto laceranti, avevano approdato alla ridefinizione e all'assestamento di nuovi rapporti all'interno di un'Europa che dal regolamento dei contrasti epocali scatenatisi nelle epoche della Riforma e Controriforma e della gigantesca lotta tra la Grande Nazione e i suoi nemici non vide messe in discussione le proprie energie complessive e quindi anche la sua capacità di dare un sempre maggiore impulso al processo di europeizzazione del mondo. Per contro la violenta contrapposizione tra il comunismo posto al servizio degli interessi del nuovo Stato sovietico e l'Europa capitalistica e borghese - scoppiata con estrema virulenza proprio in un dopoguerra in cui a occidente di Mosca si dispiegava il grande disordine generato dalle irragionevoli imposizioni delle potenze vincitrici alle potenze vinte costituì un ulteriore dirompente fattore e segnale che la centralità dell'Europa era giunta alla sua fase terminale. Sennonché la coscienza troppo spesso non è in grado di rispecchiare la realtà dei fatti: la Gran Bretagna e la Francia - in ciò rafforzate dalla scelta degli Stati Uniti di tornare all'isolazionismo e confortate e compiaciute nelle loro ambizioni imperialistiche in particolare dalla posizioni recentemente acquisite in Medio Oriente per effetto del dissolvimento dell'impero ottomano - ritennero di poter ancora erigersi a rappresentanti e tutrici di una centralità europea divenuta nei fatti ormai del tutto illusoria.

Il quinto elemento che stava a testimoniare del chiudersi della parabola che aveva visto l'Europa esercitare il ruolo preponderante nelle vicende del mondo era riconducibile al posto occupato sulla scena mondiale dagli Stati Uniti e dal Giappone. Non si trattava più, come a cavallo tra Otto e Novecento, di un iniziale 
moto ascendente, bensì di potenze consolidate in grado di strappare al Vecchio continente quello che alla vigilia della grande guerra era stato l'ultimo suo monopolio: l'assoluta superiorità militare.

Della portata degli sconvolgimenti avvenuti espresse piena consapevolezza nel 1923 il conte austriaco Coudenhove-Kalergi, che, ardente fautore di una ricostruzione federalistica dell'Europa, con uno spirito al contempo realistico e utopistico, osservava: “Oggi l'Europa non è più il centro della terra (...) Il mondo si è emancipato dall'Europa. (...) Può l'Europa nella sua frammentazione politica ed economica mantenere la sua pace e la sua indipendenza di fronte alle crescenti potenze mondiali extra-europee o essa è costretta, per conservare la propria esistenza, ad organizzarsi in federazione di Stati?”.

\section{Il tentativo dell' "Attila moderno" e la definitiva “esplosione" dell'Europa}

Il decennio seguente la pace di Versailles fu un periodo di relativa ripresa dell'Europa, assestatasi però su basi che si sarebbero rivelate assai precarie; poi sopravvenne lo sconvolgimento economico provocato dalla grande crisi che, partita nel 1929 dagli Stati Uniti, dilagò anche nel Vecchio continente con esiti devastanti. Gli anni della relativa ripresa avevano avuto due principali fondamenti: costituiti l'uno dalla preponderanza delle due potenze vincitrici, la Gran Bretagna e la Francia, e dalla capacità del capitalismo continentale di resistere all'assalto rivoluzionario guidato da Mosca rimasta infine isolata, dove Stalin aveva imposto una politica di raccoglimento nazionale; l'altro, dopo il caos economico del 1923, dalla ricostruzione dell'apparato industriale della Germania grazie al piano di investimenti americani varato nel 1924, che consentì al paese di raggiungere per alcuni anni una certa stabilità politica e istituzionale.

Quale fosse tutta l'importanza dello spostamento del centro dell'economia mondiale dal Vecchio al Nuovo Mondo fu mostrato dalla crisi del 1929. Il che in effetti era già pienamente emerso durante la guerra. Solo infatti in virtù dell'aiuto finanziario e delle forniture di mezzi prima e in seguito dell'intervento militare diretto degli Stati Uniti, Gran Bretagna, Francia e Italia erano riuscite ad aver ragione della Germania e dell'Austria-Ungheria; e sempre solo in virtù di rinnovati aiuti e del vigoroso moto ascendente che negli anni Venti aveva caratterizzato l'economia americana - giunta a detenere verso la fine del decennio oltre il 40 per cento della produzione industriale mondiale - il continente aveva potuto risalire la china. La crisi partita da Wall Street e subito dilagata in Europa fu la prova del 
nove, questa volta in chiave negativa, che il benessere o il malessere europeo dipendevano direttamente dallo stato di salute degli Stati Uniti.

Nei dieci anni che precedettero lo scoppio della seconda guerra mondiale quello che era parso un barlume di ordine europeo - l'ordine imposti dai paesi risultati vincitori nel 1918 - andò gradualmente logorandosi fino a anche nel settembre 1939 non si giunse alla vera e propria deflagrazione dell’Europa. La Germania era stata il paese più drammaticamente investito dalle conseguenze della crisi partita dall'America. La faticosamente raggiunta stabilità politica e istituzionale andò in frantumi. I partiti comunista e nazionalsocialista - nemici giurati l'uno dell'altro ma uniti dall'odio verso la repubblica nata a Weimar nel 1919, che, nonostante la loro aggressività, erano rimasti modeste minoranze in termini di consenso popolare e di rappresentanza parlamentare - dopo il 1929 andarono vertiginosamente crescendo, così da indebolire sempre più socialdemocratici, cattolici del Centro e liberaldemocratici, fino a che i nazisti guidati da Hitler, preso il sopravvento insieme sui comunisti e sui sostenitori delle istituzioni democratiche e parlamentari, nel gennaio 1933 non ascesero al potere, allargando le frontiere del fascismo internazionale, tenuto a battesimo in Italia da Mussolini nel 1922, e più in generale dei regimi autoritari che a mano a mano erano andati dilagando e sarebbero ancora dilagati nel continente. Nei sei anni che seguirono la presa del potere da parte di Hitler in Germania, la mappa geopolitica dell'Europa fu caratterizzata: dalla rapida e forte ripresa della potenza industriale e militare tedesca e dall'avvicinamento via via più stretto dell'Italia alla Germania, dalla esplicitata volontà di quest'ultima di mettere in radicale discussione mediante una politica aggressiva gli equilibri frutto della pace del 1919; dalla debolezza nei suoi confronti da parte di Gran Bretagna e Francia; dal processo di modernizzazione industriale e militare dell'Unione Sovietica, uscita in misura rilevante dalla posizione di precedente debolezza e assurta a soggetto di primo piano della politica internazionale. L'Europa fu così divisa in tre centri dominanti: quello nazi-fascista guidato da Germania e Italia e animato da intenti aggressivi ed espansionistici, quello democratico rappresentato da Gran Bretagna e Francia segnato da non secondarie differenze e da una palese arrendevolezza in politica estera, e quello costituito dall'Unione Sovietica, la quale, oltre che sull'accresciuta forza dello Stato, poteva contare sull'appoggio dei partiti comunisti europei, seppure, con l'unica rilevante eccezione del Partito comunista francese, questi fossero deboli o persino ridotti a entità marginali, perseguitati quando non cancellati dalla scena politica. L'idea e la presunzione della superiorità era tornata con tragica forza in Europa ad opera del nazionalsocialismo, sia nel razzismo che proclamava il diritto al predominio degli ariani e perseguiva l'oppressione tanto della nemica "razza ebraica", di cui auspicava l'annientamento, quanto di tutte le razze e popolazioni "inferiori” che intendeva ridurre a uno stato di soggezione semischiavistica (il veleno razzistico 
trovò un terreno fertile di diffusione in Francia, in Polonia, in altri paesi dell'Est dove aveva radici antiche - e nell'Italia fascista che nel 1938 adottò leggi razziali), sia nella convinzione propria di Hitler e dei suoi seguaci di poter riaffermare nel mondo la centralità dell'Europa mediante i successi della potenza tedesca.

L'Europa aveva accumulato un potenziale esplosivo tale per cui, quando fosse stata accesa la miccia, essa sarebbe diventata, per la seconda volta nel Novecento e in un grado molto maggiore, un cumulo di macerie. La miccia venne accesa nel settembre 1939 con l'aggressione alla Polonia da parte della Germania e la sua spartizione tra questa e l'Unione Sovietica. La guerra civile spagnola - allargatasi ad affare internazionale - aveva poco prima già documentato a qual punto fossero arrivati i contrasti intereuropei. È altamente significativo che ai tempi delle sue iniziali folgoranti vittorie militari la Germania nazista ritenne che esse ponessero le basi del ritorno, cui sopra si è accennato, alla centralità dell'Europa nel mondo. Questo sogno era nutrito dall'idea che si potesse costruire l'alleanza planetaria tra le razze superiori di cui gli ariani erano chiamati a costituire il fondamento sotto la guida di un'Europa rigenerata da "un ordine nuovo" - avente come presupposto la distruzione dell'Unione Sovietica e la riduzione degli slavi a popolo servo - alla testa del quale doveva esservi il Terzo Reich millenario. Fu questo il tentativo di unificazione dell'Europa e di rilancio del suo primato messo in atto da Hitler, che Einaudi con un'espressione pregnante avrebbe poi definito "l’Attila moderno".

Il suo fallimento provocò da un lato la drammatica “esplosione” dell'Europa, che si trovò nel 1945 in uno stato di devastazione materiale e sociale senza precedenti, dall'altro lo spostamento dello scettro della forza nelle mani del colosso americano e di quello euro-asiatico. Il fatto che ancora una volta, come già nel 1918, la Gran Bretagna e la Francia - questa disastrosamente sconfitta nel 1940 dalla Germania - si fossero trovate dalla parte dei vincitori non nascondeva il significato della loro definitiva retrocessione a potenze di secondo rango, entrate nella sfera di influenza della superpotenza occidentale e incapaci di difendersi dalla superpotenza orientale se non ponendosi sotto l'ombrello protettivo statunitense. Altro segno del crollo europeo fu che la ricostruzione economica dei paesi rimasti nell'area capitalistica fu resa possibile, in misura ancora più determinante di quanto non fosse avvenuto dopo il 1918, unicamente dall'aiuto americano. Infine, a sanzionare il declino del continente che per secoli aveva avuto saldamente nelle proprie mani le chiavi della potenza politica, economica e militare fu la doppia divisione che oppose per quasi mezzo secolo l' "Europa americana" all' “Europa sovietica” e la Germania occidentale alla Germania orientale. I paesi europei non erano più padroni dei loro destini.

La condizione di subalternità cui era giunta l'Europa era riassumibile nei seguenti punti: 1) come già nel 1914, nel 1939 suo perverso privilegio era stato 
di aver scatenato il conflitto mondiale, ma di essere stata ancora una volta incapace in grado di risolverlo; 2) a differenza della prima guerra mondiale, che aveva pur sempre lasciato Gran Bretagna e Francia nella posizione di potenze di primo livello, la seconda eliminò qualsiasi Stato integralmente europeo dai vertici del potere mondiale; 3) la forza necessaria per dare - comunque fosse - un ordine al globo divenne prerogativa degli Stati Uniti e dell'Unione Sovietica; 4) alla tradizionale rivalità fra gli Stati europei, alle loro alleanze e schieramenti contrapposti si sostituì la subordinazione dell'Europe divisa alle due superpotenze: subordinazione che volle dire per gli europei occidentali cadere sotto l'egemonia americana e per gli orientali subire un dominio totalitario.

In tal modo, con la soggezione dell'Europa resa "provincia” per una parte di una potenza extraeuropea e per l'altra di una potenza euroasiatica, si chiudeva un'epoca della storia. Nel 1945 si profilò non solo la definitiva cancellazione di quelli che ancora potevano apparire gli ultimi quanto mai parziali residui della centralità un tempo esercitata dal Vecchio continente, ma anche la perdita, più o meno radicale a seconda dei vari paesi, delle componenti fondamentali alla base della sovranità degli Stati ovvero il potere di decidere in maniera pienamente autonoma delle proprie istituzioni, delle strategie in materia economica, della politica estera, delle alleanze militari. Nel corso del quarantennio della "guerra fredda” che oppose l'Occidente capitalistico all'Oriente comunista, questa perdita accomunò entrambe le due Europe, come reso platealmente evidente dal fatto che le chiavi della guerra e della pace - elemento tanto cruciale del potere sovrano - vennero del tutto sottratte a ciascuna di esse, diventando prerogativa dei due Stati erettisi a dominatori del mondo. Tant'è che la pace interna di cui finalmente godette il Vecchio continente non fu opera degli europei stessi ma effetto degli equilibri stabiliti dalle superpotenze, le quali imposero loro margini di decisione, favorirono certi tipi di regime, ne promossero e dettarono altri, propagandarono e diffusero le loro ideologie nel quadro dei confliggenti processi di "americanizzazione" e di "sovietizzazione”. La fine poi degli imperi coloniali europei dopo il 1945, accompagnata da fallimentari tentativi di mantenerne almeno i residui e da guerre crudeli e ingloriose, volle altresì dire che le frontiere dell'Europa, un tempo tanto allargatesi all'esterno mediante il processo di "europeizzazione de mondo", tornarono a rinchiudersi entro i suoi confini propriamente geografici. 


\section{Bibliografia}

Bairoch, P 1997, Victoires et déboires. Histoire économique et sociale du monde du XVI siècle à nos jours, Gallimard, Paris.

Bracher, KD 1982, Zeit der Ideologien, Deutsche Verlags-Anstalt GmbH, Stuttgart.

Dehio, L 1948, Gleichgewicht oder Hegemonie, Scherpe-Verlag, Krefeld.

Fuller, JFC 1961, The conduct of war 1789-1961, Eyre Methuen, London.

Galasso, G 2001, Storia d'Europa, Laterza, Roma-Bari.

Hughes, HS 1958, Consciousness and Society. The Reorientation of European Social Thought, Harvard University Press, Cambridge.

Judt, T 2005, Postwar. A History of Europe Since 1945, Penguin Press, New York.

Kennedy, P 1987, The Rise and Fall of the Great Powers, Random House, New York.

Landes, DS 1998, The Wealth and Poverty of Nations, Norton, New York.

Rossi, P 2007, L'identità dell'Europa, il Mulino, Bologna.

Salvadori, ML 1990, Storia dell'età moderna e contemporanea, Loescher, Torino.

Salvadori, ML 2001, "Ascesa e crisi della centralità dell'Europa”, in La storia. I Grandi problemi dell'Età Contemporanea. 5, eds N Tranfaglia \& M Firpo, Garzanti, Milano.

Silvestri, M 1977, La decadenza dell'Europa occidentale, 1890-1939, 3 voll., Einaudi, Torino.

Villani, P 1983, Trionfo e crollo del predominio europeo, il Mulino, Bologna. 